\title{
A generalization of vortex lines
}

\author{
M. Fecko, a) \\ Department of Theoretical Physics, Comenius University in Bratislava, \\ Slovakia
}

Helmholtz theorem states that, in ideal fluid, vortex lines move with the fluid. Another Helmholtz theorem adds that strength of a vortex tube is constant along the tube. The lines may be regarded as integral surfaces of a 1-dimensional integrable distribution (given by the vorticity 2-form). In general setting of theory of integral invariants, due to Poincaré and Cartan, one can find $d$-dimensional integrable distribution (given by a possibly higher-rank form) whose integral surfaces show both properties of vortex lines: they move with (abstract) fluid and, for appropriate generalization of vortex tube, strength of the latter is constant along the tube.

PACS numbers: 02.40.-k, 45.20.Jj, 47.15ki, 47.32.C-

Keywords: vortex lines, vortex tubes, Poincaré and Cartan integral invariants, Helmholtz theorems

\section{CONTENTS}

\section{Introduction $\quad 2$}

II. Time-independent flow

A. Poincaré integral invariants

B. Stationary Euler equation 4

C. Helmholtz statement on vortex lines - stationary case 5

D. Helmholtz statement on vortex tubes - stationary case 6

III. Time-dependent flow

A. Cartan integral invariants

B. Non-stationary Euler equation 8

C. Helmholtz statement on vortex lines - general case 9

D. Helmholtz statement on vortex tubes - general case

IV. Generalization to surfaces $\quad 10$

A. Time-independent (Poincaré) case $\quad 10$

B. Time-dependent (Cartan) case

V. Conclusions $\quad 11$

Acknowledgments $\quad \lcm{12}$

$\begin{array}{ll}\text { A. Decomposition of forms } & 12\end{array}$

B. A proof of (40)

C. Dimension of the distribution $\mathcal{D}$

a) Electronic mail: fecko@fmph.uniba.sk 


\section{INTRODUCTION}

In hydrodynamics, vortex lines are field lines of vorticity vector field $\boldsymbol{\omega}$, which is curl of velocity field $\mathbf{v}$. Vortex tube is a surface made of vortex lines passing through each point of a transversal circuit (so that the circuit then encircles the tube).

It was as early as 1858 (see Ref. 1 and Refs. 2 - 6 ) that Helmholtz proved that, in the case of ideal and barotropic fluid that is only subject to conservative forces,

- vortex lines "move with the fluid" (the same fact is sometimes expressed as that the lines are "frozen into the fluid" or that "vortex lines are material lines") and that

- strength of a vortex tube is the same at all cross-sections.

Here the strength is defined as the flux of the vorticity field $\boldsymbol{\omega}$ for cross-section itself or, via Stokes theorem, as circulation of the velocity field $\mathbf{v}$ round the circuit cut by the cross-section.

Geometrical (and even topological) language has proved very effective in hydrodynamics since a long time ago. In particular, for obtaining and classifying of conserved quantities, one can use Hamiltonian structure of hydrodynamic equations or the interconnection of symmetries and conserved quantities, see e.g. Refs. $7-10$.

When treated geometrically, the Helmholtz statements may get specific meaning.

For example, Arnold succeeded to show (see Ref. 11), that the Euler equation for incompressible fluid on $n$-dimensional Riemannian manifold has an elegant formulation as the geodesic equation on the Lie group of volume-preserving diffeomorphisms of the given manifold. (In strong analogy with a much simpler - finite-dimensional - description of a rotating top, where the Lie group is $S O(3)$.) In this approach, Helmholtz theorem stems from invariance of coadjoint orbits with respect to the dynamics. (For subsequent work in this direction, see Refs. 12 16, and, in particular, monography 7).

The point of view this paper is based on starts from regarding hydrodynamics of ideal fluid as an application of the theory of integral invariants due to Poincaré and Cartan (see Refs. 17 and 18, Ref. 19 or, in modern presentation, Ref. 20 23). Then, original Poincaré version of the theory refers to stationary (time-independent) flow, described by stationary Euler equation, whereas Cartan's extension embodies the full, possibly time-dependent, situation.

Let us remark that although integral invariants due to Poincaré and Cartan are mostly known from classical Hamiltonian mechanics, see e.g. Ref. 24, its realm of applications is wider (see Refs. 18 and 19).

The idea of a proof of Helmholtz theorem on vortex lines might go, within the integral invariants setting, as follows (for details, see below). First, vortex lines are identified with integral surfaces of an 1-dimensional integrable distribution, defined in terms of an appropriate 2-form. Second, structure of the (Euler) equation of motion immediately reveals that the 2-form is Lie-invariant w.r.t. the flow of the fluid. So, third, the corresponding distribution is invariant w.r.t. the flow and, consequently, its integral surfaces are invariant w.r.t. the flow of the fluid. But this is exactly what Helmholtz statement says.

Now, it turns out that the same reasoning may be repeated within the general integral invariant setting (so beyond even the " $n$-dimensional Riemannian hydrodynamics"). What differs is that we have an integrable distribution based on a possibly higher-degree Lieinvariant differential form, there. In particular, the distribution may be higher-dimensional and, consequently, its integral surfaces become then higher-dimensional, too. Nevertheless, they still obey the Helmholtz-like rule of "moving with the fluid" (i.e. the abstract flow in the general theory translates the integral surfaces into one another).

Concerning the vortex tubes Helmholtz theorem, proof of the original statement is very easy and corresponding generalization to integral invariants setting is almost self-evident.

The structure of the paper is as follows.

In Section 【A, in order to make the text self-contained, we shortly remind the reader, in modern language, of the Poincaré theory of integral invariants. Then, in Section IIB, we present stationary Euler equation rewritten in a form needed for profiting from the Poincaré theory. Sections 【C and ID then show how (easily) one obtains Helmholtz results within 
this scheme.

The same program is then repeated, for the case of time-dependent Euler equation (based on Cartan's extension of the theory of integral invariants), in Sections III A. III B. IIIC and IIID.

Finally, as the principal topic of the paper, general, possibly higher-dimensional surfaces moving with the (abstract) fluid in the phase space of a system, are studied in Sections IV A (stationary case) andIVB (time-dependent case; here also extended phase space plays a role).

\section{TIME-INDEPENDENT FLOW}

\section{A. Poincaré integral invariants}

Consider a manifold $M$ endowed with dynamics given by a vector field $v$

$$
\dot{\gamma}=v \quad \dot{x}^{i}=v^{i}(x)
$$

The field $v$ generates the dynamics (time evolution) via its flow $\Phi_{t} \leftrightarrow v$. We will call the structure phase space

$$
\left(M, \Phi_{t} \leftrightarrow v\right) \quad \text { phase space }
$$

In this situation, let us have a $k$-form $\alpha$ and consider its integrals over various $k$-chains ( $k$-dimensional surfaces) $c$ on $M$. Due to the flow $\Phi_{t}$ corresponding to $v$, the $k$-chains flow away, $c \mapsto \Phi_{t}(c)$. Compare the value of integral of $\alpha$ over the original $c$ and integral over $\Phi_{t}(c)$. If, for any chain $c$, the two integrals are equal, it reflects a remarkable property of the form $\alpha$ with respect to the field $v$. We call it integral invariant:

$$
\int_{\Phi_{t}(c)} \alpha=\int_{c} \alpha \Leftrightarrow \int_{c} \alpha \text { is integral invariant }
$$

For infinitesimal $t \equiv \epsilon$ we have

$$
\int_{\Phi_{\epsilon}(c)} \alpha=\int_{c} \alpha+\epsilon \int_{c} \mathcal{L}_{v} \alpha
$$

(plus, of course, higher order terms in $\epsilon$; here $\mathcal{L}_{v}$ is Lie derivative along $v$ ). Since (3) is to be true for each $c$, we get from (4)

$$
\mathcal{L}_{v} \alpha=0
$$

This is the differential version of the statement (3).

In specific situations, it may be enough that some integral only behaves invariantly when restricted to an important sub-class of $k$-chains, namely $k$-cycles. These are chains whose boundary vanish:

$$
\partial c=0 \quad c=\text { cycle }
$$

If this is the case, the condition (5) is overly strong. It can be weakened to

$$
\mathcal{L}_{v} \alpha=d \tilde{\beta}
$$

for some form $\tilde{\beta}$. (The form $\mathcal{L}_{v} \alpha$ may just be exact rather than vanish.) Indeed, in one direction, Eqs. (6) and (7) then give

$$
\int_{c} \mathcal{L}_{v} \alpha=\int_{c} d \tilde{\beta}=\int_{\partial c} \tilde{\beta}=0
$$


so that (3) is fulfilled. In the opposite direction, if (3) is to be true for each cycle, the form under the last integral sign in (44) is to be exact due to de Rham theorem, so (77) holds.

According to whether the integrals of forms are invariant for arbitrary $k$-chains or just for $k$-cycles, integral invariants are known as either absolute invariants (for any $k$-chain) or relative ones (just for $k$-cycles; notice that $\mathcal{L}_{v}(d \alpha)=0$ holds from (7), so whenever $\alpha$ gives relative invariant, $d \alpha$ already gives an absolute one).

Now, let us see what we can say about relative integral invariants. The condition (7) may be rewritten, using Cartan's formula

$$
i_{v} d+d i_{v}=\mathcal{L}_{v}
$$

as

$$
i_{v} d \alpha=d \beta
$$

(where $\beta=\tilde{\beta}-i_{v} \alpha$ ). Therefore the following main statement on relative invariants is true:

$$
i_{v} d \alpha=d \beta \quad \Leftrightarrow \quad \oint_{c} \alpha=\text { relative invariant }
$$

So we can identify the presence of relative integral invariant in differential version: on phase space $(M, v)$, we find a form $\alpha$ such that the l.h.s. of Eq. (10) is exact.

\section{B. Stationary Euler equation}

Euler equation for ideal (inviscid) fluid

$$
\rho\left(\partial_{t} \mathbf{v}+(\mathbf{v} \cdot \boldsymbol{\nabla}) \mathbf{v}\right)=-\nabla p-\rho \nabla \Phi
$$

(see, e.g. Ref. 4 and 25) reduces, for stationary flow, to

$$
(\mathbf{v} \cdot \nabla) \mathbf{v}=-\frac{1}{\rho} \nabla p-\nabla \Phi
$$

Here mass density $\rho$, velocity field $\mathbf{v}$, pressure $p$ and potential $\Phi$ of the volume force field are functions of $\mathbf{r}$.

In general, equation of state of the fluid may be written as

$$
p=p(\rho, s) \quad \text { general fluid }
$$

where $s$ is (specific) entropy (i.e. entropy per unit mass). However, one can think of an important model, where the pressure depends on $\rho$ alone:

$$
p=p(\rho) \quad \text { barotropic fluid }
$$

In this case, there exists $P(\mathbf{r})$, called specific enthalpy, such that

$$
\frac{1}{\rho} \nabla p=\nabla P
$$

and (13) takes the form

$$
(\mathbf{v} \cdot \boldsymbol{\nabla}) \mathbf{v}=-\boldsymbol{\nabla}(P+\Phi)
$$

Now it turns out (check in Cartesian coordinates) that Eq. (17) may be rewritten in the form of Eq. (10) for the particular choice $\alpha=\tilde{v}$ and $\beta=-\mathcal{E}$, i.e. as

$$
i_{v} d \tilde{v}=-d \mathcal{E} \quad \text { Euler equation }
$$


(stationary and barotropic), where

$$
\tilde{v}:=\mathbf{v} \cdot d \mathbf{r} \quad\left(\equiv b_{g} v \equiv g(v, \cdot)\right)
$$

is the covector $\left(=1\right.$-form) associated with the velocity vector field $v=v^{i} \partial_{i}$ in terms of "lowering of index" (三 $b_{g}$ procedure) and

$$
\mathcal{E}:=v^{2} / 2+P+\Phi \quad \text { Bernoulli function }
$$

The vorticity 2-form $d \tilde{v}$, present in Eq. (18), is of crucial importance for us. We have

$$
\begin{aligned}
\tilde{v} & =\mathbf{v} \cdot d \mathbf{r} \\
d \tilde{v} & =(\operatorname{curl} \mathbf{v}) \cdot d \mathbf{S} \equiv \boldsymbol{\omega} \cdot d \mathbf{S} \\
i_{\gamma^{\prime}} d \tilde{v} & =\left(\boldsymbol{\omega} \times \mathbf{r}^{\prime}\right) \cdot d \mathbf{r}
\end{aligned}
$$

(see, e.g. $\$ 8.5$ in Ref. 26) so that, first, $d \tilde{v}$ indeed encodes complete information about vorticity vector field $\boldsymbol{\omega}$ and, second, the equation

$$
i_{\gamma^{\prime}} d \tilde{v}=0 \quad \text { vortex line equation }
$$

expresses the fact that $\gamma(\lambda) \leftrightarrow \mathbf{r}(\lambda)$ corresponds to vortex line (the prime symbolizes tangent vector w.r.t. parameter $\lambda$; particular parametrization is, however, irrelevant).

The form (18) of the Euler equation turns out to be very convenient. Short illustration:

1. Application of $i_{v}$ on both sides gives

$$
v \mathcal{E}=0 \quad \text { Bernoulli equation }
$$

(saying that $\mathcal{E}$ is constant along streamlines).

2. Application of $i_{\gamma^{\prime}}$ on both sides (where $\gamma^{\prime}$ is from (24)) gives

$$
\gamma^{\prime} \mathcal{E}=0
$$

(saying that $\mathcal{E}$ is constant along vortex-lines).

3. Putting $d \tilde{v}=0$ (irrotational flow) leads to

$$
\mathcal{E}=\text { const }
$$

(a version of Bernoulli equation saying that $\mathcal{E}$ is, then, constant throughout the fluid).

4. Just looking at (11), (18) and (19) one obtains

$$
\oint_{c} \mathbf{v} \cdot d \mathbf{r}=\text { const. } \quad \text { Kelvin's theorem }
$$

(velocity circulation is conserved quantity).

5. Application of $d$ on both sides gives Helmholtz theorem (see the next Section \C).

\section{Helmholtz statement on vortex lines - stationary case}

Application of $d$ on both sides of (18) and using (9) results in

$$
\mathcal{L}_{v}(d \tilde{v})=0
$$

This is, however, nothing but infinitesimal version of the statement

$$
\Phi_{t}^{*}(d \tilde{v})=d \tilde{v} \quad \Phi_{t} \leftrightarrow v
$$

or, in words, that the vorticity 2 -form $d \tilde{v}$ is invariant w.r.t. the flow of the fluid. 


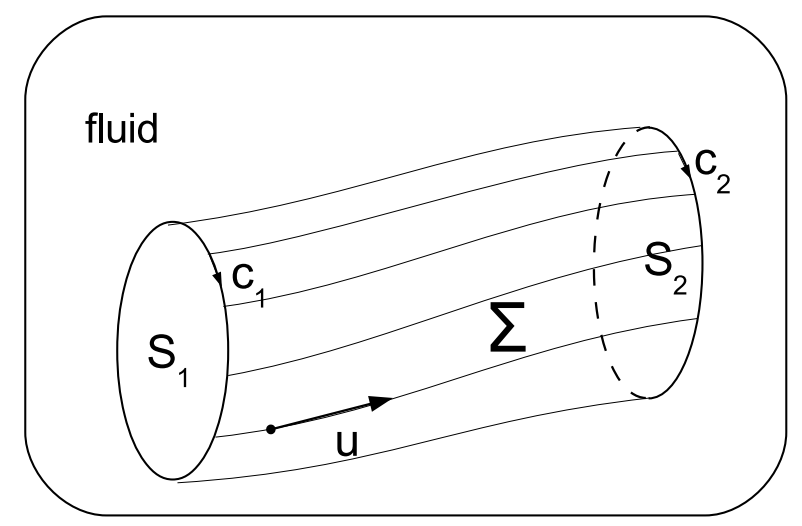

FIG. 1. Vortex tube $\Sigma$ is made of vortex lines emanating from (all points of) circuit $c_{1}=\partial S_{1}$ and entering the circuit $c_{2}=\partial S_{2}$. Equation (35) says that strength (vorticity flux) for the cross-section $S_{1}$ is the same as the strength for the cross-section $S_{2}$.

Now, we can define a distribution $\mathcal{D}$ in terms of $d \tilde{v}$ :

$$
\mathcal{D}:=\left\{\text { vectors } w \text { such that } i_{w} d \tilde{v}=0 \text { holds }\right\}
$$

Due to Frobenius criterion the distribution is integrable. Indeed, let $w_{1}, w_{2} \in \mathcal{D}$. Then, because of the identity

$$
i_{\left[w_{1}, w_{2}\right]}=\left[\mathcal{L}_{w_{1}}, i_{w_{2}}\right] \equiv \mathcal{L}_{w_{1}} i_{w_{2}}-i_{w_{2}} \mathcal{L}_{w_{1}}
$$

(see, e.g., Ch.5.Ex.21 in Ref. 27 or $\$ 6.2$ in Ref. 26) plus (9) one immediately sees that

$$
i_{\left[w_{1}, w_{2}\right]} d \tilde{v}=0
$$

i.e. $\left[w_{1}, w_{2}\right] \in \mathcal{D}$, too. So $\mathcal{D}$ is integrable.

From (23) and (24) we see that the distribution is 1-dimensional (in those points where $\boldsymbol{\omega} \neq 0$ ) and that its integral surfaces are exactly vortex lines. But this means that Helmholtz statement is true: because of (30) and (31) the distribution $\mathcal{D}$ is invariant w.r.t. $\Phi_{t} \leftrightarrow v$ and, consequently, its integral surfaces (i.e. vortex lines) are invariant w.r.t. $\Phi_{t} \leftrightarrow v$, too.

\section{Helmholtz statement on vortex tubes - stationary case}

This statement is purely kinematical, it concerns the concept of vorticity itself. It holds for arbitrary velocity fields $v$, even those which do not satisfy equations of motion (so they cannot occur).

Let $u$ be a vector field defined by $i_{u} d \tilde{v}=0$, i.e. a field tangent, at each point, to the vortex line passing through the point (see Eq. (24)). Notice that any vortex line may be created from its single point by the flow $\Phi_{s}$ of $u$ and the same holds (using evident freedom $u \mapsto f u$, $f$ being a function) for the vortex tube bounded by fixed circuits $c_{1}$ and $c_{2}$ (boundaries of fixed cross-sections $S_{1}$ and $S_{2}$, see Fig. (1).

Consider the artificial (!) "dynamics" given by $u$. Then the equation $i_{u} d \tilde{v}=0$ may be regarded as a particular case of the basic equation (11) from the general theory of Poincaré integral invariants (with $v \mapsto u, \alpha \mapsto \tilde{v}$ and $\beta \mapsto 0$ ). So,

$$
\oint_{c} \tilde{v} \equiv \oint_{c} \mathbf{v} \cdot d \mathbf{r} \quad \text { is relative invariant }
$$


and, consequently,

$$
\int_{S} d \tilde{v} \equiv \oint_{S} \boldsymbol{\omega} \cdot d \mathbf{S} \quad \text { is absolute invariant }
$$

both of them w.r.t. our "artificial dynamics" generated by $u$ (as opposed to the real dynamics generated by the fluid velocity field $v$ ). Then, however, Eq. (35) exactly says that the vorticity flux does not depend on particular choice of cross-section $S$ cutting the tube.

Alternatively, one can use the proof of Eq. (40) given in Appendix B (with $\xi \mapsto u$ and $\sigma \mapsto \tilde{v})$

\section{TIME-DEPENDENT FLOW}

\section{A. Cartan integral invariants}

Cartan proposed, as a first step, to study the dynamics given in (1) and (2) on $M \times \mathbb{R}$ (extended phase space; time coordinate is added) rather than on $M$. Using the natural projection

$$
\pi: M \times \mathbb{R} \rightarrow M \quad(m, t) \mapsto m \quad\left(x^{i}, t\right) \mapsto x^{i}
$$

the forms $\alpha$ and $\beta$ (from Poincaré theory) may be pulled-back from $M$ onto $M \times \mathbb{R}$ and then combined into a single $k$-form

$$
\sigma=\hat{\alpha}+d t \wedge \hat{\beta}
$$

(Here, we denote $\hat{\alpha}=\pi^{*} \alpha$ and $\hat{\beta}=\pi^{*} \beta$ ). In a similar way, define a vector field

$$
\xi=\partial_{t}+v
$$

Its flow clearly consists of the flow $\Phi_{t} \leftrightarrow v$ on the $M$ factor combined with the trivial lapsing of time in the $\mathbb{R}$ factor. Now a simple check (for which Appendix $\mathbb{A}$ might come in handy) reveals that the equation

$$
i_{\xi} d \sigma=0
$$

is equivalent to (10). And the main statement (11) takes the form

$$
i_{\xi} d \sigma=0 \quad \Leftrightarrow \quad \oint_{c} \sigma=\text { relative invariant }
$$

Here the meaning of the r.h.s. of (40) is as follows: take a cycle $c_{1}$ located in the hyper-plane $t=t_{1}$ and its image $c_{2}$ w.r.t. the flow of $\xi$ (it is located in the hyper-plane $t=t_{2}$ ). Then integrals of $\sigma$ over $c_{1}$ and $c_{2}$ give the same number. (Notice that $d t \wedge \hat{\beta}$ part of $\sigma$ does not contribute, since $d t$ vanishes on the hyper-planes.) So, indeed, statements (11) and (40) are, in this interpretation, equivalent.

First new result by Cartan (w.r.t. Poincaré) is an observation that more general interpretation of (40) is possible. Namely, take any two cycles in $M \times \mathbb{R}$ which encircle common tube of solutions (here "solutions" mean integral curves of $\xi$, i.e. solutions of the dynamics as seen from $M \times \mathbb{R}$ ). Then, still, integrals of $\sigma$ over $c_{1}$ and $c_{2}$ give the same number. See a proof in Appendix B.

Further Cartan's generalization, however, is much more interesting for us. Recall that (37) might also be regarded as a decomposition of the most general $k$-form $\sigma$ on $M \times \mathbb{R}$, see Appendix $\AA$. In this case, $\hat{\alpha}$ and $\hat{\beta}$ need not be obtained by pull-back from $M$. Rather, 
they are the most general spatial forms on $M \times \mathbb{R}$. One easily sees that, in comparison with just pull-backs, they may be time-dependent, i.e. it may happen that

$$
\mathcal{L}_{\partial_{t}} \hat{\alpha} \neq 0 \quad \mathcal{L}_{\partial_{t}} \hat{\beta} \neq 0
$$

(In coordinate presentation, their components may depend on time.)

Recall that the proof of (40) from Appendix B did not use any details of the decomposition. The structure of the equation (39) is all one needs. Notice, however, that the equivalence of (39) and (10) is no longer true when (41) holds. Instead, one easily computes (with the help of Appendix $\mathrm{A}$ ) that

$$
i_{\xi} d \sigma=0 \quad \Leftrightarrow \quad \mathcal{L}_{\partial_{t}} \hat{\alpha}+i_{v} \hat{d} \hat{\alpha}=\hat{d} \hat{\beta}
$$

(the term $\mathcal{L}_{\partial_{t}} \hat{\alpha}$ is new). So, the equation

$$
\mathcal{L}_{\partial_{t}} \hat{\alpha}+i_{v} \hat{d} \hat{\alpha}=\hat{d} \hat{\beta}
$$

is the equation that time-dependent forms $\hat{\alpha}$ and $\hat{\beta}$ are to satisfy in order that integral of $\sigma$ is to be a relative integral invariant.

\section{B. Non-stationary Euler equation}

Let us retell Cartan's results from the last section in the context of hydrodynamics, i.e. for particular choice (see Eq. (18))

$$
\sigma=\hat{v}-\mathcal{E} d t
$$

where, in usual coordinates $(\mathbf{r}, t)$ on $E^{3} \times \mathbb{R}$,

$$
\hat{v}:=\mathbf{v} \cdot d \mathbf{r} \equiv \mathbf{v}(\mathbf{r}, t) \cdot d \mathbf{r}
$$

From (42) we get

$$
i_{\xi} d \sigma=0 \quad \Leftrightarrow \quad \mathcal{L}_{\partial_{t}} \hat{v}+i_{v} \hat{d} \hat{v}=-\hat{d} \mathcal{E}
$$

One easily checks (e.g. in Cartesian coordinates $(\mathbf{r}, t))$ that

$$
\mathcal{L}_{\partial_{t}} \hat{v}+i_{v} \hat{d} \hat{v}=-\hat{d} \mathcal{E}
$$

is nothing but the complete, time-dependent, Euler equation (12). Therefore the timedependent Euler equation may also be written in remarkably succinct form as

$$
i_{\xi} d \sigma=0 \quad \text { Euler equation }
$$

The form (48) of the Euler equation turns out to be very convenient for analyzing some of its cosequences. Two examples:

1. Just looking at (40), (48) and (44) one obtains

$$
\oint_{c} \mathbf{v} \cdot d \mathbf{r}=\text { const. } \quad \text { Kelvin's theorem }
$$

(the two loops $c_{1}$ and $c_{2}$ are usually in constant-time hyper-planes $t=t_{1}$ and $t=t_{2}$ ).

2. Application of $d$ on both sides gives very quickly Helmholtz theorem (see the next Section IIIC). 


\section{Helmholtz statement on vortex lines - general case}

Application of $d$ on both sides of (48) and using formula (9) results in

$$
\mathcal{L}_{\xi}(d \sigma)=0
$$

This is, however, nothing but infinitesimal version of the statement

$$
\Phi_{t}^{*}(d \sigma)=d \sigma \quad \Phi_{t} \leftrightarrow \xi
$$

or, in words, that the $d \sigma$ is invariant w.r.t. the flow of the fluid (regarded as the flow of $\xi$ on $M \times \mathbb{R})$.

Now, we want to see an integrable distribution behind vortex lines, again. Define the distribution $\mathcal{D}$ in terms of annihilation of as many as two exact forms:

$$
\mathcal{D} \leftrightarrow \quad i_{w} d \sigma=0=i_{w} d t
$$

By repeating the reasoning from (32) and (33) one concludes that $\mathcal{D}$ is integrable.

The distribution $\mathcal{D}$ is, however, also invariant w.r.t. the flow of the fluid. (Because of (50) and the trivial fact that $\mathcal{L}_{\xi}(d t)=0$.) So, integral submanifolds (surfaces) move with the fluid.

What do they look like? Although perhaps not visible at first sight, they are nothing but vortex lines.

Indeed, making use of general formula (A3) from Appendix A and the form (47) of Euler equation we can write

$$
\begin{aligned}
d \sigma & =\hat{d} \hat{v}+d t \wedge\left(\mathcal{L}_{\partial_{t}} \hat{v}+\hat{d} \mathcal{E}\right) & & \text { always } \\
& =\hat{d} \hat{v}+d t \wedge\left(-i_{v} \hat{d} \hat{v}\right) & & \text { on solutions }
\end{aligned}
$$

Let us now contemplate Eq. (52). It says, that the distribution consists of spatial vectors (i.e. those with vanishing time component, therefore annihilating $d t$ ) which, in addition, annihilate $d \sigma$.

Let $w$ be arbitrary spatial vector. Denote, for a while, $i_{w} \hat{d} \hat{v}=: \hat{b}$ (it is a spatial 1 -form). Then, from (54),

$$
i_{w} d \sigma=\hat{b}-d t \wedge i_{v} \hat{b}
$$

from which immediately

$$
i_{w}(d \sigma)=0 \quad \Leftrightarrow \quad \hat{b} \equiv i_{w} \hat{d} \hat{v}=0
$$

This says that we can, alternatively, describe the distribution $\mathcal{D}$ as consisting of those spatial vectors which, in addition, annihilate $\hat{d} \hat{v}$ (rather than $d \sigma$, as it is expressed in the definition (52)). But Eqs. (45) and (22) show that

$$
\hat{d} \hat{v}=\boldsymbol{\omega} \cdot d \mathbf{S} \equiv \boldsymbol{\omega}(\mathbf{r}, t) \cdot d \mathbf{S}
$$

so that $\hat{d} \hat{v}$ is nothing but the vorticity 2-form and, therefore, the integral surfaces of $\mathcal{D}$ may indeed be identified with vortex lines. So, Helmholtz statement is also true in the general, time-dependent, case. (Notice that the system of vortex lines looks, in general, different in different times. This is because its generating object, the vorticity 2 -form $\hat{d} \hat{v}$, depends on time.)

\section{Helmholtz statement on vortex tubes - general case}

Vortex tube is a genuinely spatial concept and the statement concerns purely kinematical property of any velocity field at a single time (see the beginning of Sec. IID). So, no (change of) dynamics has any influence on it. If the statement were true before, it remains to be true now. 


\section{GENERALIZATION TO SURFACES}

In this section we present details concerning the surfaces mentioned in the Introduction. By now it is easy, since we already know all the relevant ideas from hydrodynamics parts.

All symbols which occur here refer to objects mentioned in the general theory of integral invariants (due to Poincaré and Cartan, respectively, i.e. objects from Sections IIA and IIIA) rather than to their special instances used in hydrodynamics (including the $n$ dimensional case).

\section{A. Time-independent (Poincaré) case}

We apply $d$ on both sides of (10) or (7) and get

$$
\mathcal{L}_{v}(d \alpha)=0
$$

So, the $(k+1)$-form $d \alpha$ is invariant w.r.t. the flow generated on $M$ by $v$.

Now, define a distribution $\mathcal{D}$ given by annihilation of the form $d \alpha$ :

$$
\mathcal{D}:=\left\{\text { vectors } w \text { such that } i_{w} d \alpha=0 \text { holds }\right\}
$$

Its dimension is therefore

$$
\begin{aligned}
\operatorname{dim} \mathcal{D} & =\operatorname{dim} M-\operatorname{rank} d \alpha \\
& \leq \operatorname{dim} M-(k+1)
\end{aligned}
$$

(if $\alpha$ is $k$-form, see Appendix $\mathrm{C}$, the rank of $d \alpha$ is expected to be constant).

The distribution $\mathcal{D}$ has the following two properties.

First, it is invariant w.r.t. the flow generated on $M$ by $v$. (This is because of (58).)

Second, with the help of (32) and (9) we see that

$$
i_{w_{1}} d \alpha=0=i_{w_{2}} d \alpha \quad \Rightarrow \quad i_{\left[w_{1}, w_{2}\right]} d \alpha=0
$$

i.e.

$$
w_{1}, w_{2} \in \mathcal{D} \quad \Rightarrow \quad\left[w_{1}, w_{2}\right] \in \mathcal{D}
$$

So, due to Frobenius criterion, $\mathcal{D}$ is integrable.

Put the two properties together, this means that integral surfaces (submanifolds) of the distribution move with the (abstract) fluid, exactly in the spirit of the Helmholtz theorem on vortex lines. (Notice that this behavior equally holds for any surface of smaller dimension which resides within the maximal-dimension one.)

Now consider a vector field $W \in \mathcal{D}$ (so it satisfies $i_{W} d \alpha=0$; this is analog of the field $w$ directed along vortex lines, discussed in Sec. [ID). Application of its flow $\Phi_{s}$ on $k$-dimensional boundary $c_{1} \equiv \partial S_{1}$ of a transversal $(k+1)$-dimensional surface $S_{1}$ gives a $(k+1)$-dimensional analog of vortex tube, $\Sigma$ (see Fig 22). So

$$
\partial \Sigma=c_{1}-c_{2}
$$

Repeating either the reasoning from Appendix B or that from Sec. IID we show that the (analog of the) strength of the tube is constant along the tube

$$
\int_{S_{1}} d \alpha=\int_{S_{2}} d \alpha
$$

This is an analog of Helmholtz theorem on vortex tubes. 


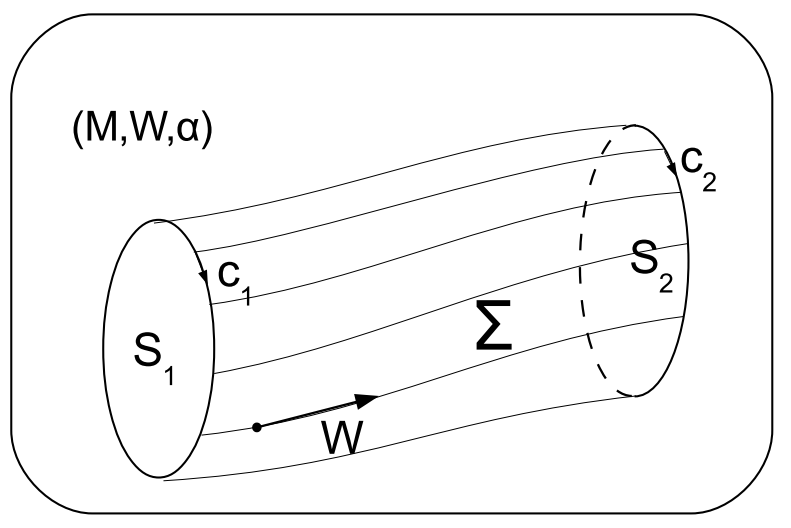

FIG. 2. Higher-dimensional analog of vortex tube, $\Sigma$. It is bounded by a $k$-dimensional boundary $c_{1}$ of a transversal $(k+1)$-dimensional surface $S_{1}$ from the left and similarly by $c_{2} \equiv \partial S_{2}$ from the right. Here $S_{2} \equiv \Phi_{s}\left(S_{1}\right)$ for some $s$.

\section{B. Time-dependent (Cartan) case}

We apply $d$ on both sides of (39) and get

$$
\mathcal{L}_{\xi}(d \sigma)=0
$$

So, the $(k+1)$-form $d \sigma$ is invariant w.r.t. the flow generated on $M \times \mathbb{R}$ by $\xi$.

Now, define a distribution $\mathcal{D}$ given by spatial vectors which annihilate the form $d \sigma$ :

$$
\mathcal{D}:=\left\{\text { spatial vectors } w \text { such that } i_{w} d \sigma=0\right\}
$$

Put another way, it is defined as

$$
w \in \mathcal{D} \quad \Leftrightarrow \quad i_{w} d \sigma=0=i_{w} d t
$$

The distribution is invariant w.r.t. the flow generated on $M \times \mathbb{R}$ by $\xi$ (since both its generating forms, $d \sigma$ as well as $d t$, are Lie-invariant w.r.t. $\xi$ ).

In addition, due to Frobenius criterion, the distribution is integrable. (One just applies (62) to both $d \sigma$ and $d t$.)

Put the two properties together, this means that integral submanifolds (surfaces) of the distribution move with the (abstract) fluid in the spirit of the Helmholtz theorem on vortex lines.

Finally, notice that, on solutions of Eq. (39), the distribution $\mathcal{D}$ generated by the pair of forms $(d \sigma, d t)$ coincides with that generated by the pair $(\hat{d} \hat{\alpha}, d t)$. (Just repeat argumentation in (53) - (56) replacing $\hat{v} \mapsto \hat{\alpha}, \mathcal{E} \mapsto-\hat{\beta}$ and Eq. (47) $\mapsto$ Eq. (43).) So it consists of spatial vectors annihilating $\hat{d} \hat{\alpha}$. Therefore, the statement about surfaces moving with the (abstract) fluid here, in Sec. IVB, is a natural generalization (namely to time-dependent flow) of the corresponding statement mentioned in Sec. IV A

Concerning the "vortex tube" Helmholtz theorem, it has nothing to do with dynamics and therefore it is trivially true also here (see Sec. 【IID).

\section{v. CONCLUSIONS}

The main point discussed in this paper is a statement concerning the general setting of the theory of integral invariants (rather than the "ideal hydrodynamics on Riemanian 
manifolds" or "higher-dimensional hydrodynamics" discussed, e.g., in Ref. 7 and in the numerous papers mentioned in references therein).

Namely, in the theory of integral invariants, both the time-independent version of Poincaré and the extended, time-dependent version of Cartan, one can find specific surfaces which move with the (abstract) "fluid".

When the theory is applied to 3D-hydrodynamics of ideal and barotropic fluid only subject to potential force, the surfaces become 1-dimensional and reduce to well-known and useful concept of vortex lines. Their property of moving with the fluid (now the real one) becomes the celebrated Helmholtz theorem from 1858.

So, in this sense, the surfaces may be regarded as a generalization of the vortex lines.

One can also define, in the general higher-dimensional case, an analog of the hydrodynamical concept of vortex tubes and check that (an analog of) Helmholtz theorem on strength of the tubes is still true.

\section{ACKNOWLEDGMENTS}

I acknowledge support from grant VEGA 1/0985/16.

\section{Appendix A: Decomposition of forms}

On $M \times \mathbb{R}$, a $p$-form $\alpha$ may be uniquely decomposed as

$$
\alpha=d t \wedge \hat{s}+\hat{r}
$$

where both $\hat{s}$ and $\hat{r}$ are spatial, i.e. they do not contain the factor $d t$ in its coordinate presentation (here, we assume adapted coordinates, $t$ on $\mathbb{R}$ and some $x^{i}$ on $M$ ). Simply, after writing the form in coordinates, one groups together all terms which do contain $d t$ once and, similarly, terms which do not contain $d t$ at all. Note, however, that $t$ still can enter components of any (even spatial) form. Therefore, when performing exterior derivative $d$ of a spatial form, say $\hat{r}$, there is a part, $\hat{d} \hat{r}$, which does not take into account the $t$-dependance of the components (if any; as if it was performed just on $M$ ), plus a part which, on the contrary, only operates on the $t$ variable. Putting both parts together, we have

$$
d \hat{r}=d t \wedge \mathcal{L}_{\partial_{t}} \hat{r}+\hat{d} \hat{r}
$$

Then, for a general form (A1), we get

$$
d \alpha=d t \wedge\left(-\hat{d} \hat{s}+\mathcal{L}_{\partial_{t}} \hat{r}\right)+\hat{d} \hat{r}
$$

\section{Appendix B: A proof of (40)}

The proof is amazingly simple (see $\$ 44$ of Ref. 20). Consider integral of $d \sigma$ over the $(k+1)$-chain $\Sigma$ given by the family of trajectories (solutions) connecting $c_{1}$ and $c_{2}$ (so that $\partial \Sigma=c_{1}-c_{2}$, see Fig. 3, Then

$$
\begin{aligned}
\int_{\Sigma} d \sigma & \stackrel{\text {.. }}{=} \int_{\partial \Sigma} \sigma=\oint_{c_{1}} \sigma-\oint_{c_{2}} \sigma \\
& \stackrel{\text {.. }}{=} 0
\end{aligned}
$$

The second line (zero) comes from observation, that $\xi$ is tangent to $\Sigma$ by construction, so that integral of $d \sigma$ over $\Sigma$ consists of infinitesimal contributions proportional to $d \sigma(\xi, \ldots)$, all of them vanishing because of (39). 


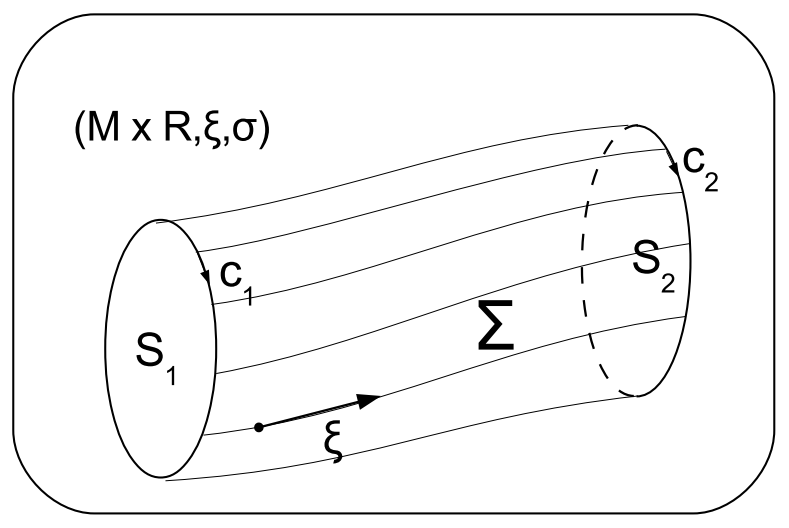

FIG. 3. $\Sigma$ is given by the family of trajectories (solutions) connecting $c_{1}$ and $c_{2}$, so that $\partial \Sigma=c_{1}-c_{2}$. Integral of $d \sigma$ over $\Sigma$ vanishes, since $\xi$ is tangent to $\Sigma$ and annihilates $d \sigma$.

\section{Appendix C: Dimension of the distribution $\mathcal{D}$}

The distribution $\mathcal{D}$ from Eq. (59) is given as the kernel of the linear map

$$
f: w \mapsto i_{w} d \alpha
$$

Rank of the form $d \alpha$ is, by definition, the dimension of the image space of the map (C1). Then, due to the standard rank-nullity theorem applied to $f$, we have

$$
\operatorname{dim} \operatorname{Ker} f+\operatorname{dim} \operatorname{Im} f=\operatorname{dim} T_{x} M \equiv \operatorname{dim} M
$$

or

$$
\operatorname{dim} \mathcal{D}+\operatorname{rank} d \alpha=\operatorname{dim} M
$$

So, (60) holds.

Now rank of a $p$-form is at least $p$ (it is $p$ for decomposable form), so

$$
\operatorname{rank} d \alpha \geq k+1
$$

and, therefore, (61) holds.

The dimension of $\mathcal{D}$ in time-dependent case (i.e. given by (67) or (68) ) equals the dimension of $\mathcal{D}$ from the time-independent case (given by (59) ). Indeed, as is mentioned in the last paragraph of Sec. IVB, the distribution $\mathcal{D}$ on $M \times \mathbb{R}$ generated by the pair of forms $(d \sigma, d t)$ coincides, on solutions, with that generated by the pair $(\hat{d} \hat{\alpha}, d t)$. So it consists of spatial vectors annihilating $\hat{d} \hat{\alpha}$. Or, when thinking of dimensions alone, of vectors on $M$ annihilating $d \alpha$.

${ }^{1}$ H. Helmholtz, Journal für die reine und angewandte Mathematik 55, 25-55 (1858).

${ }^{2}$ C. Truesdell, The Kinematics of Vorticity (Indiana University Press, 1954).

${ }^{3}$ P. Saffman, Vortex Dynamics (Cambridge University Press, 1992).

${ }^{4}$ G. K. Batchelor, An Introduction to Fluid Dynamics (Cambridge University Press, 2002).

${ }^{5}$ J. Z. Wu, H. Y. Ma, and M.D.Zhou, Vorticity and Vortex Dynamics (Springer-Verlag Berlin Heidelberg, 2006).

${ }^{6}$ K. S. Thorne and R. D. Blandford, Modern Classical Physics: Optics, Fluids, Plasmas, Elasticity, Relativity, and Statistical Physics (Princeton University Press, 2017).

${ }^{7}$ V. Arnold and B. Khesin, Topological Methods in Hydrodynamics (Springer-Verlag, 1998). 
${ }^{8}$ S. C. Anco, J. Math. Fluid Mech. 15, 439-451 (2013), arXiv:1209.4251 [math-ph]

${ }^{9}$ S. C. Anco, A. Dar, and N. Tufail, Proc.Roy.Soc. A 471, 20150223 (2015), arXiv:1503.08859 [math-ph]

${ }^{10}$ N. Besse and U. Frisch, J. Fluid Mech. 825, 4129-478 (2017), arXiv:1701.01592v1 [physics.flu-dyn]

${ }^{11}$ V. I. Arnold, Ann. Inst. Fourier 16, 319-361 (1966).

${ }^{12}$ V. Guillemin and S. Sternberg, Ann. Phys. 127, 220-253 (1980).

${ }^{13}$ J. Marsden and A. Weinstein, Physica D 7, 305-323 (1983).

${ }^{14}$ J. Marsden, T. Ratiu, and A. Weinstein, Trans.Am.Math.Soc. 281, 147-177 (1984).

${ }^{15}$ S. P. Novikov, Rus. Math. Surv. 37, 1-56 (1982).

${ }^{16}$ B. A. Khesin and Y. V. Chekanov, Physica D 40, 119-131 (1989).

${ }^{17} \mathrm{H}$. Poincaré, Les Méthodes nouvelles de la Mécanique Céleste, (III, Invatiants intégraux) (GauthierVillars et fils, 1899).

${ }^{18}$ E. Cartan, Lecons sur les invariants intégraux (Hermann, 1922).

${ }^{19}$ F. Gantmacher, Lectures in Analytical Mechanics (Mir, 1975).

${ }^{20} \mathrm{~V}$. Arnold, Mathematical Methods of Classical Mechanics (Springer-Verlag, 1989).

${ }^{21}$ P. Libermann and C.-M. Marle, Symplectic Geometry and Analytical Mechanics (D.Reidel, 1987).

${ }^{22}$ R. M. Kiehn, International Journal of Engineering Science 13, 941-949 (1975).

${ }^{23}$ M. Fecko, Acta Physica Slovaca 63, 261-359 (2013), arXiv:1406.0078 [physics.flu-dyn].

${ }^{24}$ L. D. Landau and E. M. Lifshitz, Mechanics (Butterworth-Heinemann Ltd., 3rd ed., 1995).

${ }^{25}$ L. D. Landau and E. M. Lifshitz, Fluid Mechanics (Pergamon Press, Second Edition, 1987).

${ }^{26}$ M. Fecko, Differential Geometry and Lie Groups for Physicists (Cambridge University Press, 2006).

${ }^{27}$ M. Crampin and F. A. E. Pirani, Applicable Differential Geometry (Cambridge University Press, 1986). 